
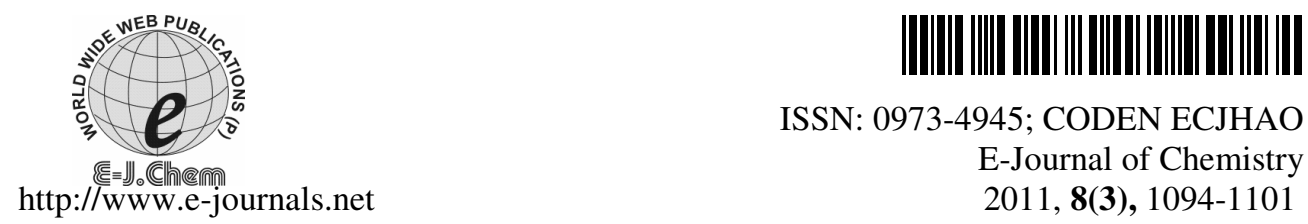

ISSN: 0973-4945; CODEN ECJHAO

E-Journal of Chemistry

2011, 8(3), 1094-1101

\title{
Ultrasonic Studies on Binary Mixtures of Acetyl Acetone at $301 \mathrm{~K}$
}

\author{
SHAIK BABU and A RADHAKRISHNA MURTHY \\ Basic Science Department \\ Chalapathi Institute of Engineering and Technology, Guntur, India \\ babu.computers@gmail.com
}

Received 28 November 2010; Accepted 22 January 2011

\begin{abstract}
The ultrasonic velocity (U), density ( $\rho)$ and viscosity ( $\eta$ ) measurements have been carried out for the binary mixtures of acetyl acetone with benzene, carbon tetra chloride and isoamyl alcohol at $301 \mathrm{~K}$. From the measured values of ultrasonic velocity, density and viscosity, parameters such as internal pressure $\left(\pi_{\mathrm{i}}\right)$, free volume $\left(V_{f}\right)$ and acoustical parameters such as adiabatic compressibility $(\beta)$, inter molecular free length $\left(L_{f}\right)$, acoustic impedance $(Z)$, relaxation time $(\tau)$ have been calculated. The results have been analyzed and interpreted in terms of molecular interactions.
\end{abstract}

Keywords: Adiabatic compressibility, Free volume, Binary mixtures, Relaxation time, Internal pressure, Free length.

\section{Introduction}

Ultrasonic methods find extensive applications for characterizing aspects of physicochemical behaviour such as the nature of molecular interactions in pure liquids as well as liquid mixtures. The thermodynamic functions of binary liquid mixtures provide insight into the structure breaking and making effect of the component liquids. Derived parameters such as internal pressure and free volume studies will be useful to know the molecular interactions of the systems. Ultrasonic velocity is one of the important parameters, frequently used to investigate intermolecular interactions in binary liquid mixtures ${ }^{1-4}$. Ultrasonic velocity measurements have been successfully employed to detect and assess weak and strong molecular interactions, present in binary ${ }^{5,6}$ and ternary ${ }^{7,8}$ liquid mixtures. In this paper, an attempt to investigate the ultrasonic studies of acetyl acetone in benzene, carbon tetrachloride, isoamyl alcohol binary liquid mixture systems at $301 \mathrm{~K}$ are made. We have measured density $(\rho)$, ultrasonic velocity $(U)$, viscosity $(\eta)$ of mixtures of acetyl acetone + benzene, + carbon tetra chloride, + isoamyl alcohol with different mole fractions at a temperature of $301 \mathrm{~K}$. From this data, acoustical parameters like adiabatic 
compressibility $(\beta)$, Inter molecular free length $\left(L_{f}\right)$, Acoustic impedance $(Z)$, Relaxation time $(\tau)$ are computed. Results are used to explain the nature of molecular interactions between mixing compounds.

\section{Experimental}

All the chemicals used in present work were analytical reagent (AR) grade (99.9\% pure) and were supplied by SD fine chemicals Ltd, Bombay, India and Qualigens chemicals, India. The liquids were thoroughly distilled to remove dissolved impurities using standard chemical procedures ${ }^{9}$. The purity of the samples was checked by the density measurements and the results were compared with the literature values. Ultrasonic velocities were measured with ultrasonic interferometer (model F80) supplied by Mittal enterprises, New Delhi, operating at a frequency of $2 \mathrm{MHz}$. It has an accuracy of $\pm 0.1 \%$. Viscosities of pure compounds and their mixtures were determined using Ostwald's viscometer with an accuracy of $\pm 0.002 \%$, calibrated with double distilled water. The densities of pure compounds and their solutions were measured accurately using $10 \mathrm{~mL}$ specific gravity bottles in Dhona electric balance precisely and the accuracy in weighing is $\pm 0.1 \mathrm{mg}$. Acoustic parameters such as adiabatic compressibility $(\beta)$, acoustic impedance (Z), relaxation time $(\tau)$, free length $\left(L_{f}\right)$, internal pressure $\left(\pi_{\mathrm{i}}\right)$ and free volume $\left(V_{f}\right)$ were determined using the following relations ${ }^{10}$.

$$
\begin{aligned}
\beta & =\frac{1}{U^{2} \rho} \\
L_{f} & =K_{T} \beta^{1 / 2} \\
V_{f} & =\left[\frac{M_{e f f} U}{\eta K}\right]^{3 / 2} \\
\pi_{i} & =b R T\left[\frac{K \eta}{U}\right]\left[\frac{\rho^{2 / 3}}{\left(M_{e f f}\right)^{7 / 6}}\right] \\
\mathrm{Z} & =\rho \mathrm{U} \\
\tau & =\frac{4 \eta}{3 \rho U^{2}}
\end{aligned}
$$

Where $\mathrm{K}_{\mathrm{T}}$ is the temperature dependent constant having a value of $199.53 \times 10^{-8}$ in MKS system, $\mathrm{K}$ is temperature independent constant whose value is $4.28 \times 10^{9}$ in MKS system, $\mathrm{b}$ is a factor depending on packing pattern which is $1.78, \mathrm{R}$ is the gas constant and $\mathrm{T}$ is temperature in $K, \eta$ is the viscosity in pascals, $U$ is ultrasonic velocity in $\mathrm{m} / \mathrm{s}, \rho$ is the density in $\mathrm{Kg} / \mathrm{m}^{3}$ and $\mathrm{M}_{\text {eff }}$ is the effective molecular weight, given by $\mathrm{M}_{\text {eff }}=\Sigma \mathrm{x}_{\mathrm{i}} \mathrm{m}_{\mathrm{i}}$ where $\mathrm{x}$ is the mole fraction and $\mathrm{m}$ is the molecular weight of the $\mathrm{i}^{\text {th }}$ component.

\section{Results and Discussion}

The binary mixture systems taken up for the present study are: acetyl acetone + benzene (I), acetyl acetone + carbon tetra chloride (II) and acetyl acetone + isoamyl alcohol (III). The experimentally determined values of velocity, density and viscosity along with the calculated values of internal pressure $\left(\pi_{\mathrm{i}}\right)$, free volume $\left(V_{f}\right)$, adiabatic compressibility $(\beta)$, free length $\left(L_{f}\right)$, acoustic impedance $(\mathrm{Z})$ and relaxation time $(\tau)$ for all the systems at $301 \mathrm{~K}$ are reported in Table 1 . 
Table 1. Velocity $(\mathrm{U})$, density $(\rho)$, viscosity $(\eta)$, internal pressure $\left(\pi_{\mathrm{i}}\right)$, free volume $\left(V_{f}\right)$, adiabatic compressibility $\left(\beta_{\mathrm{s}}\right)$, free length $\left(L_{f}\right)$, acoustic impedance $(\mathrm{Z})$ and relaxation time $(\tau)$ of binary liquid systems I, II and III at $301 \mathrm{~K}$.

System I: Acetyl acetone + benzene

\begin{tabular}{|c|c|c|c|c|c|c|c|c|c|}
\hline $\bar{x}$ & 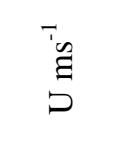 & 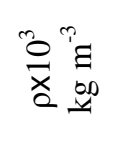 & $\stackrel{\sigma}{E}$ & 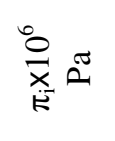 & 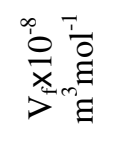 & 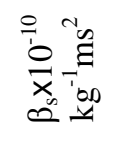 & 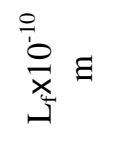 & 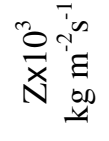 & $\underset{\frac{1}{x}}{0}$ is \\
\hline 0.0000 & 1260.0 & 0.8722 & 0.6167 & 3.6399 & 0.2277 & 7.2217 & 0.5362 & 1099.0 & 113.8651 \\
\hline 0.0797 & 1262.1 & 0.8737 & 0.6188 & 3.5575 & 0.2347 & 7.1865 & 0.5348 & 1112.6 & 114.8223 \\
\hline 0.1631 & 1264.6 & 0.8930 & 0.6224 & 3.5230 & 0.2413 & 7.0897 & 0.5312 & 1123.8 & 118.4158 \\
\hline 0.2505 & 1266.3 & 0.8950 & 0.6248 & 3.4370 & 0.2491 & 6.9712 & 0.5268 & 1133.1 & 119.5044 \\
\hline 0.3421 & 1268.0 & 0.9052 & 0.6373 & 3.3984 & 0.2511 & 6.8709 & 0.5230 & 1147.8 & 123.6801 \\
\hline 0.4382 & 1270.5 & 0.9143 & 0.6505 & 3.3579 & 0.2533 & 6.7808 & 0.5195 & 1161.2 & 127.9090 \\
\hline 0.5392 & 1272.4 & 0.9183 & 0.6604 & 3.2926 & 0.2577 & 6.7174 & 0.5171 & 1172.1 & 130.8520 \\
\hline 0.6454 & 1274.7 & 0.9346 & 0.6735 & 3.2629 & 0.2593 & 6.5922 & 0.5122 & 1190.7 & 136.2377 \\
\hline
\end{tabular}

System II: Acetyl acetone + carbon tetra chloride

\begin{tabular}{|c|c|c|c|c|c|c|c|c|c|}
\hline $\bar{x}$ & '气 & 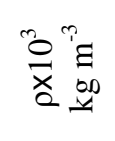 & $=\tilde{\alpha}$ & 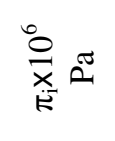 & 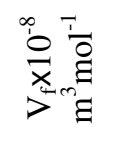 & 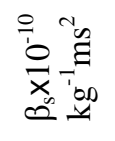 & $\stackrel{0}{\underset{0}{x}} \Xi$ & 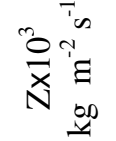 & $\frac{\sqrt[7]{0}}{x}$ in \\
\hline 0.0000 & 893.0 & 1.6279 & 1.0416 & 3.8620 & 0.1710 & 7.7031 & 0.5538 & 1453.7 & 180.2896 \\
\hline 0.1458 & 931.7 & 1.6259 & 1.0415 & 3.8013 & 0.1725 & 7.0852 & 0.5311 & 1514.8 & 196.0021 \\
\hline 0.2626 & 970.4 & 1.6188 & 1.0257 & 3.7196 & 0.1736 & 6.5598 & 0.5110 & 1570.9 & 208.4877 \\
\hline 0.3790 & 1009.1 & 1.6137 & 1.0095 & 3.6628 & 0.1740 & 6.0853 & 0.4922 & 1628.4 & 221.1971 \\
\hline 0.4870 & 1047.8 & 1.6107 & 0.9995 & 3.6092 & 0.1758 & 5.6548 & 0.4745 & 1687.7 & 235.6723 \\
\hline 0.5875 & 1086.5 & 1.6026 & 0.9989 & 3.5069 & 0.1762 & 5.2857 & 0.4587 & 1741.2 & 251.9730 \\
\hline 0.6811 & 1125.2 & 1.5945 & 0.9831 & 3.4165 & 0.1775 & 4.9534 & 0.4441 & 1794.1 & 264.6328 \\
\hline 0.7687 & 1163.9 & 1.5783 & 0.9752 & 3.3675 & 0.1788 & 4.6771 & 0.4315 & 1836.9 & 278.0058 \\
\hline
\end{tabular}

System III: Acetyl acetone + isoamyl alcohol

\begin{tabular}{|c|c|c|c|c|c|c|c|c|c|}
\hline $\bar{x}$ & '气 & 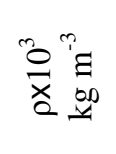 & $\stackrel{\tilde{E}}{\stackrel{\sigma}{E}}$ & 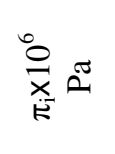 & 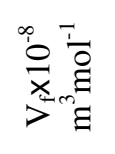 & 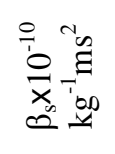 & 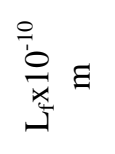 & 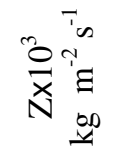 & $\frac{7}{0} \underset{x}{x}$ i \\
\hline 0.0000 & 1210.0 & 0.8109 & 3.0606 & 6.8408 & 0.0232 & 8.4224 & 0.5791 & 981.2 & 484.5138 \\
\hline 0.0891 & 1217.1 & 0.8291 & 2.0079 & 5.5376 & 0.0449 & 8.1426 & 0.5694 & 1009.1 & 328.7995 \\
\hline 0.1803 & 1224.4 & 0.8565 & 1.4125 & 4.6579 & 0.0781 & 7.7925 & 0.5570 & 1048.4 & 241.6944 \\
\hline 0.2739 & 1231.3 & 0.8663 & 1.2912 & 4.4074 & 0.0919 & 7.6588 & 0.5522 & 1060.6 & 224.7999 \\
\hline 0.3698 & 1238.7 & 0.8758 & 1.1636 & 4.1449 & 0.1103 & 7.4497 & 0.5446 & 1084.2 & 208.2728 \\
\hline 0.4681 & 1245.0 & 0.8879 & 1.0650 & 3.9333 & 0.1295 & 7.2653 & 0.5378 & 1105.5 & 195.4477 \\
\hline 0.5690 & 1252.8 & 0.9042 & 0.9274 & 3.6540 & 0.1639 & 7.0554 & 0.5300 & 1132.0 & 175.2680 \\
\hline 0.6725 & 1259.2 & 0.9205 & 0.8847 & 3.5487 & 0.1809 & 6.8532 & 0.5223 & 1158.9 & 172.1346 \\
\hline
\end{tabular}


Figure 1-6 represents the variation of internal pressure $\left(\pi_{\mathrm{i}}\right)$, free volume $\left(V_{f}\right)$, adiabatic compressibility $(\beta)$, free length $\left(L_{f}\right)$, acoustic impedance $(Z)$ and relaxation time $(\tau)$ with mole fraction for all the systems I, II and III respectively.

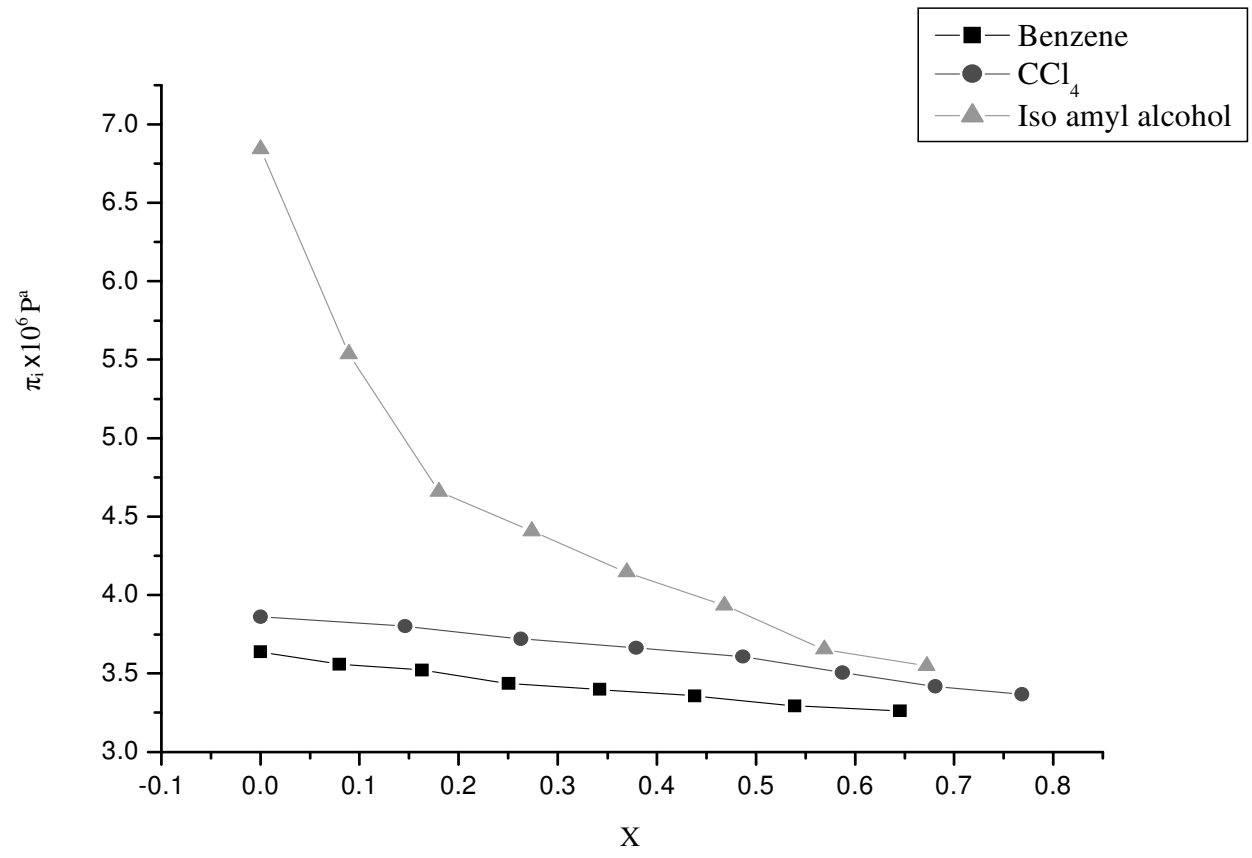

Figure 1. Variation of internal pressure $\left(\pi_{\mathrm{i}}\right)$, with mole fraction for all the systems

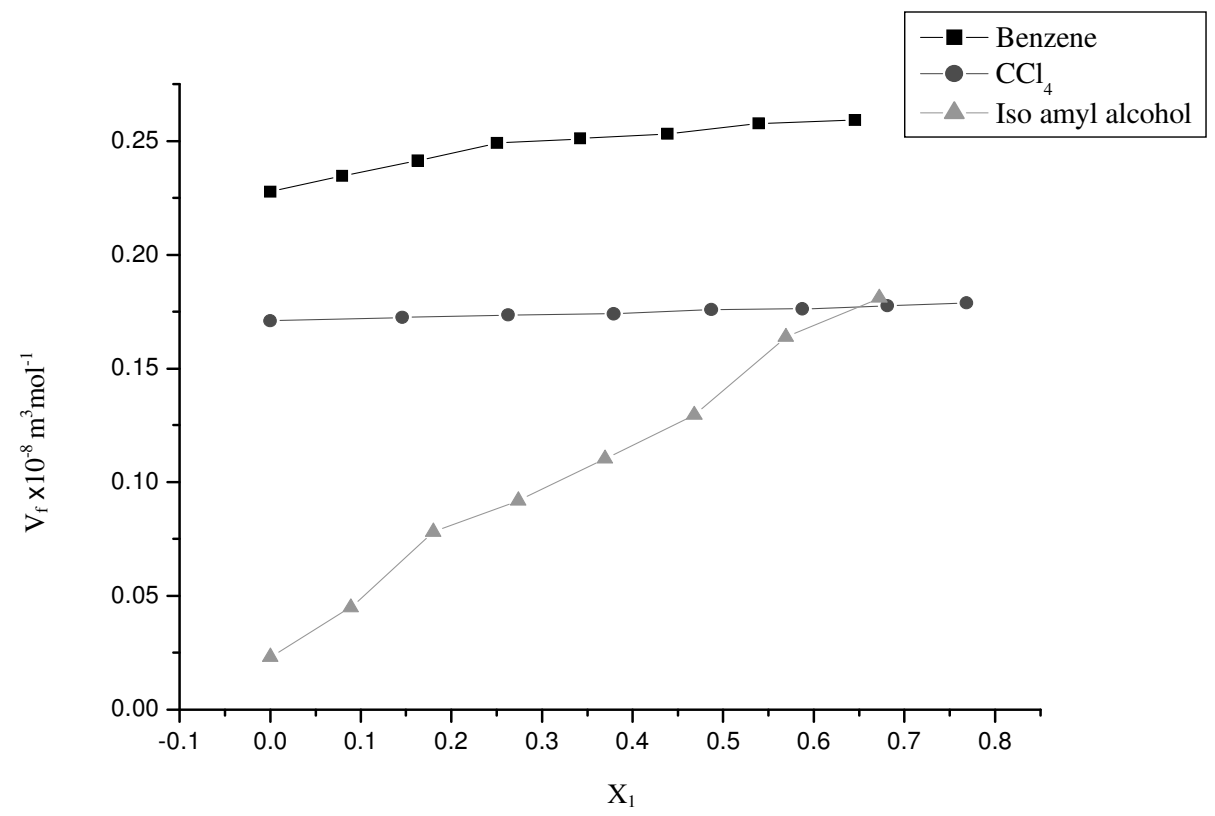

Figure 2. Variation of free volume $\left(V_{f}\right)$, with mole fraction for all the systems 


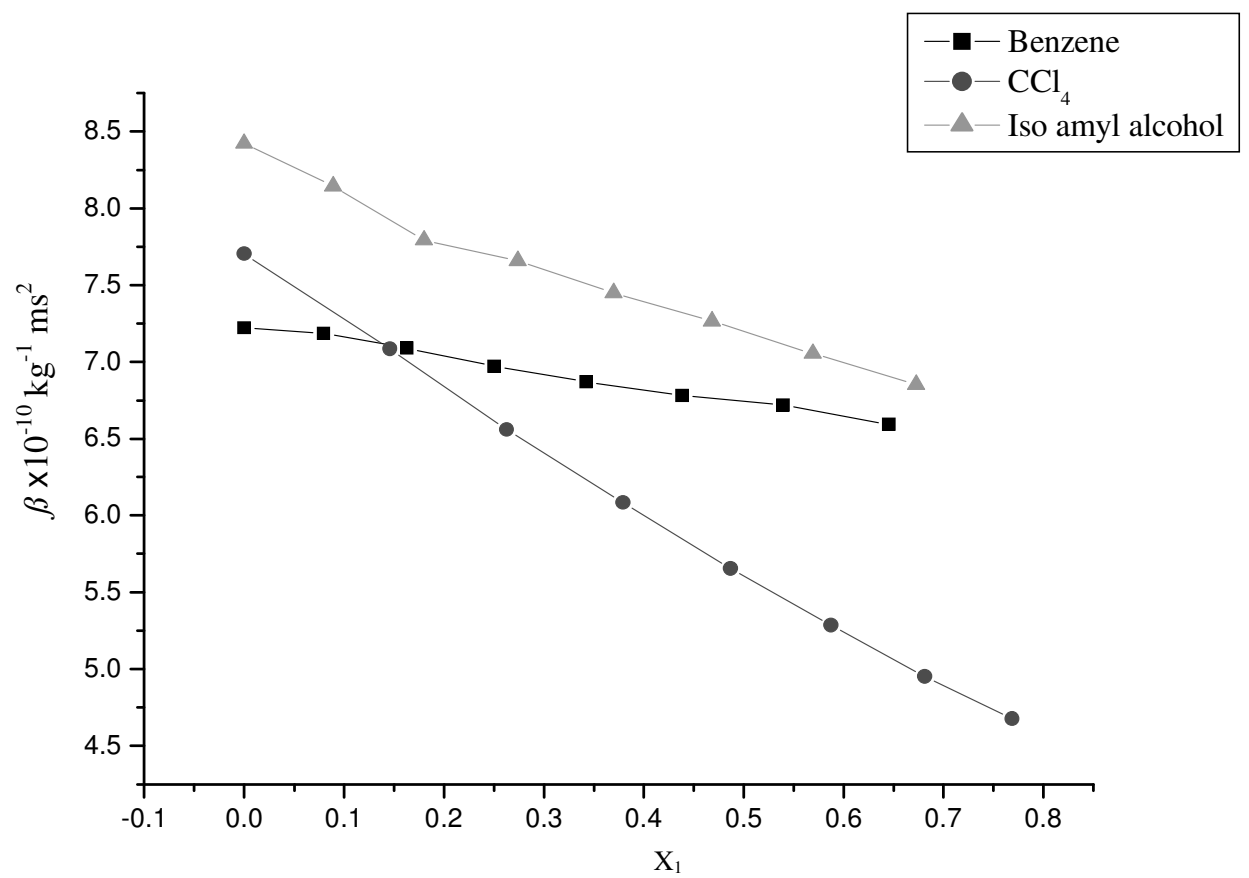

Figure 3. Variation of adiabatic compressibility $(\beta)$, with mole fraction for all the systems

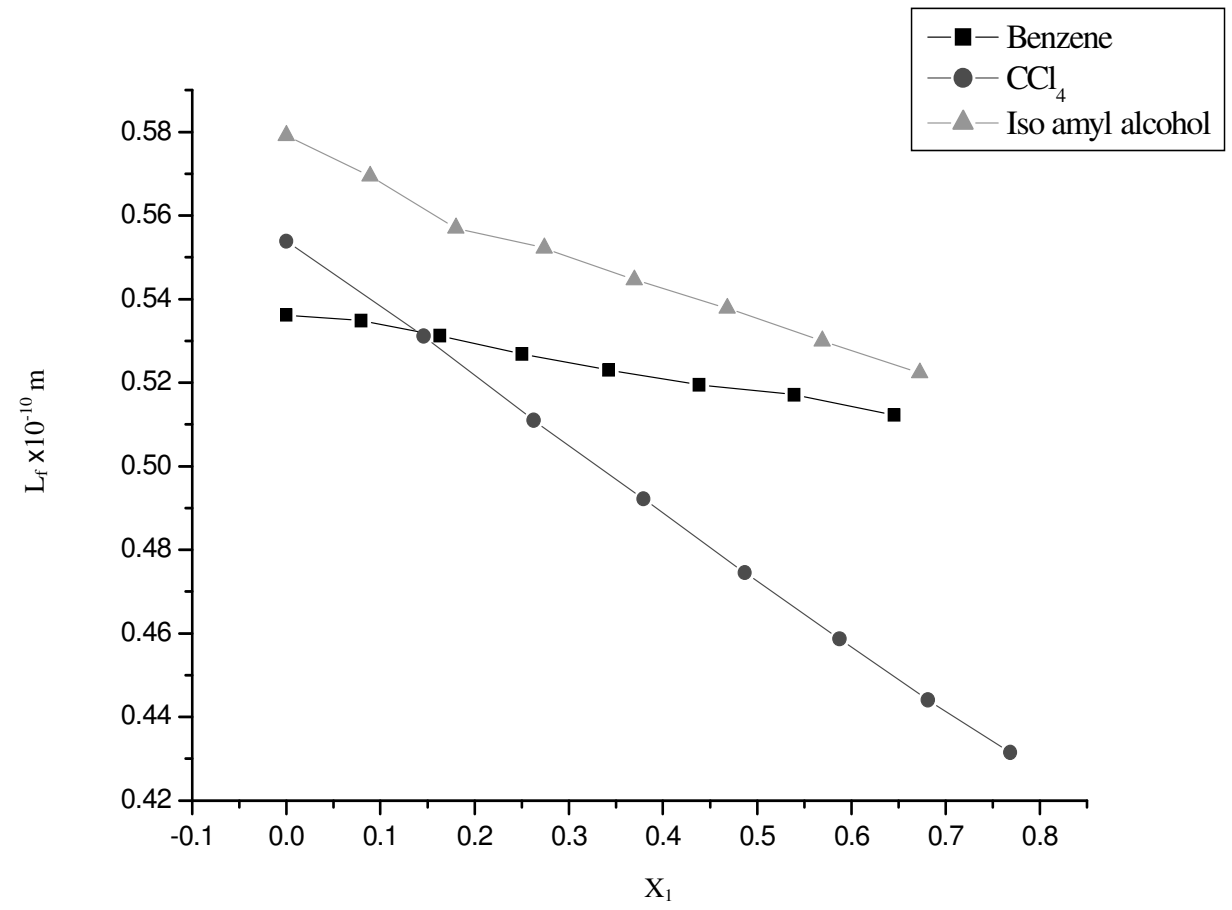

Figure 4. Variation of free length $\left(L_{f}\right)$, with mole fraction for all the systems 


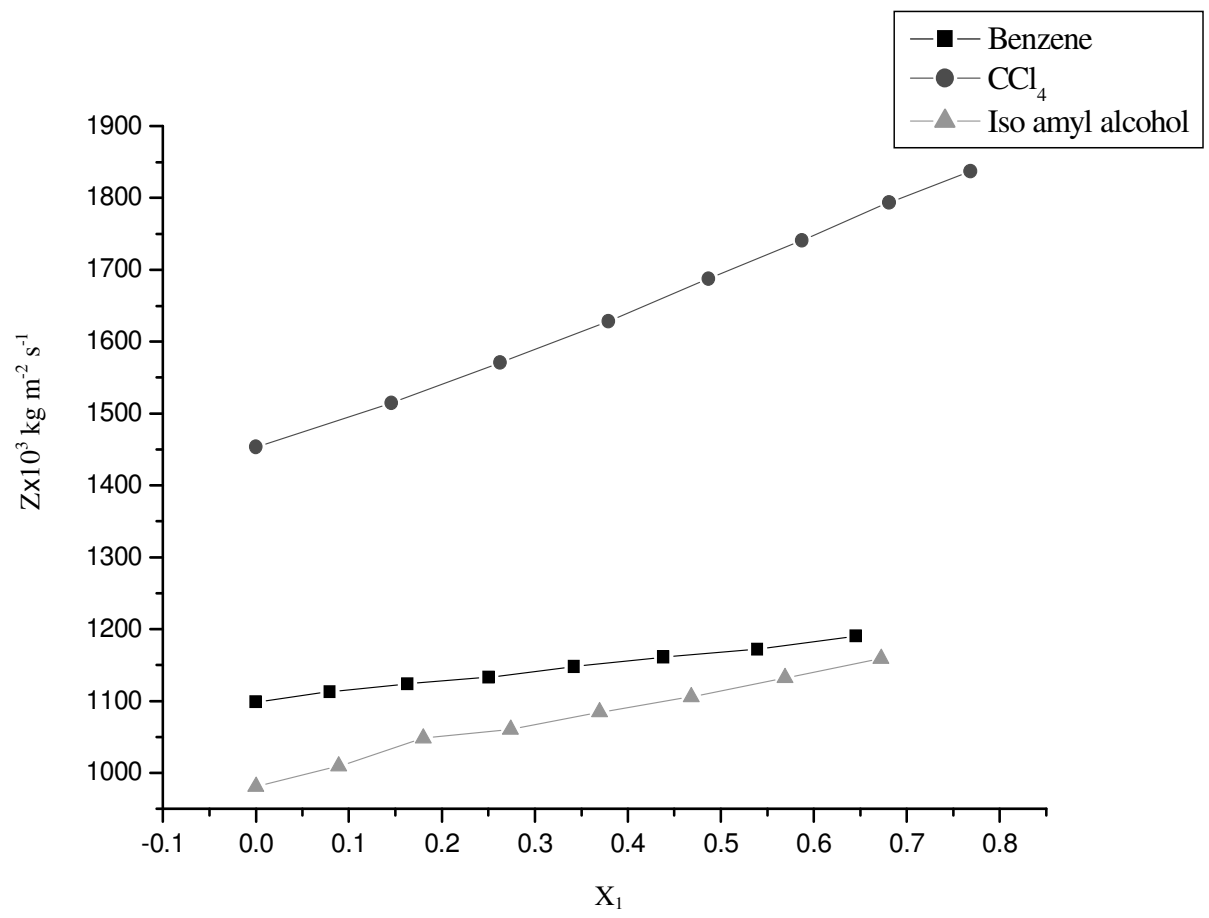

Figure 5. Variation of acoustic impedance (Z), with mole fraction for all the systems

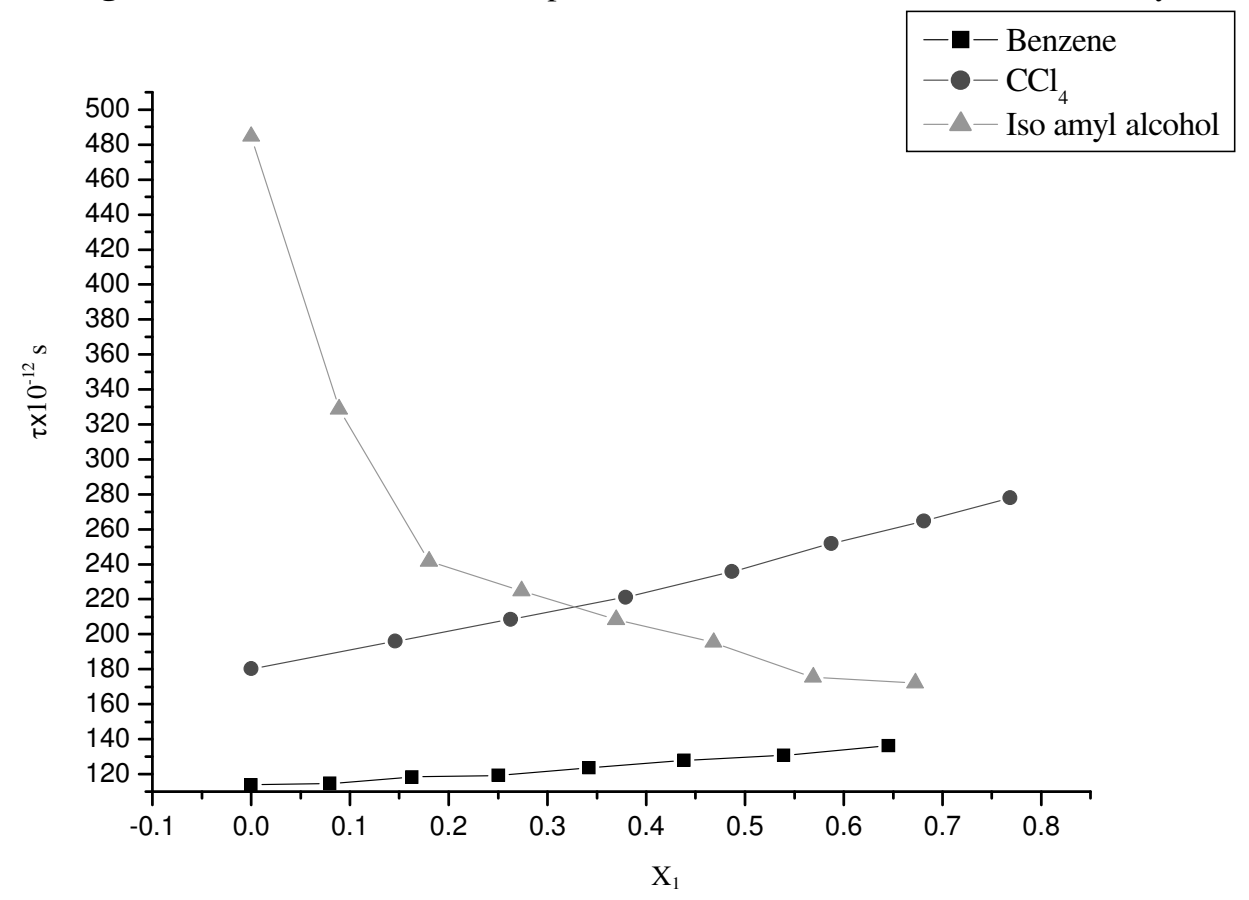

Figure 6. Variation of relaxation time $(\tau)$, with mole fraction for all the systems 
In all the three binary systems, the ultrasonic velocity increases with increasing concentration of acetyl acetone. The variation of ultrasonic velocity in a solution depends upon the increase or decrease of intermolecular free length after mixing the components. Based on the model for sound propagation proposed by eyring and kincaid ${ }^{11}$, ultrasonic velocity should increase, if the inter molecular free length decreases and vice verse. This fact was noticed in the present study for all the three binary liquid systems. Same trend was noticed by earlier workers ${ }^{12}$ in their liquid mixtures.

In system I, benzene is a non polar liquid and as the concentration of acetyl acetone increases there will be induced polarization in the benzene molecule and this result in molecular association. In fact, the molecular association increases ultrasonic velocity (U) and acoustic impedance $(Z)$, decreases intermolecular free length $\left(L_{f}\right)$ and adiabatic compressibility $(\beta)$. A reduction in adiabatic compressibility $(\beta)$ is an indication that component molecules are held close to each other. The decrease in the values of adiabatic compressibility $\left(\beta_{\mathrm{s}}\right)$ and inter molecular free length $\left(L_{f}\right)$ with increase in ultrasonic velocity $(\mathrm{U})$ further strengthens the strong molecular association between the unlike molecules through hydrogen bonding.

In system II, carbon tetra chloride is a non polar liquid and the parameters are expected to show the same trend as in system I. This fact is reflected clearly from the values of ultrasonic velocity $(\mathrm{U})$, intermolecular free length $\left(L_{f}\right)$, adiabatic compressibility $\left(\beta_{\mathrm{s}}\right)$ and acoustic impedance $(Z)$ for system II.

Alcohols usually exist in polymeric form. In the presence of polar molecules, they dissociate into monomers. In system III, both the components are polar and there is tendency for internal hydrogen bonding in acetyl acetone. With the increase of isoamyl alcohol, the tendency for intermolecular hydrogen bonding increases. The association is stronger and we expect a larger variation in the parameters. Eventually ultrasonic velocities (U) of both the components are nearly equal. In system III, this fact is reflected in increase of ultrasonic velocity $(\mathrm{U})$ and hence decrease in inter molecular free length $\left(\mathrm{L}_{f}\right)$ also it can be observed that adiabatic compressibility $(\beta)$ values decrease with increasing concentrations of isoamyl alcohol. Similar results were obtained by earlier workers ${ }^{12-14}$ in their liquid mixtures.

Acoustic impedance $(\mathrm{Z})$ of a material is the opposition exerted by the medium to displacement of the medium's particles by the sound energy. It is important to measure acoustic impedance because studies have shown that in solvent mixtures when molecular interactions occurs, acoustic impedance exhibits a non-linear variation with increasing mole fraction of solute. It has been found that the acoustic impedance gives a peak in conjunction with ultrasonic velocity maxima and isentropic compressibility minima. This was used as an essential tool to predict molecular level interactions in binary and ternary liquid mixtures. In systems I and III, Acoustic impedance $(Z)$ showed increasing trend while it showed decreasing trend in system II.

The parameter relaxation time $(\tau)$ was increasing in system I and II while it showed a decreasing trend in system III. The relaxation time of binary liquid mixtures is always much greater than either of the polar solutes in the inert solvent ${ }^{15}$.

Further, the increase in free volume $\left(V_{f}\right)$ and decrease in internal pressure $\left(\pi_{\mathrm{i}}\right)$ with rise in concentration of acetyl acetone in all the three systems under study, clearly indicate the increasing magnitude of interactions ${ }^{12,16}$.

\section{Conclusion}

In system I inter molecular association is weak. In system II and III, the molecular associations are stronger due to inter molecular hydrogen bonding. 


\section{References}

1. Murali Krishna P, Ranjit kumar B, Sathyanarayana B, Amara Jyothi K and Satyanarayana N, Indian J Pure Appl Phys., 2009, 47, 576-581.

2. Ranjit kumar B, Satyanarayana B, Asra Banu S, Amara Jyothi K, T Savitha Jyostna and Satyanarayana N, Indian J Pure Appl Phys., 2009, 47, 511.

3. Thiyagarajan R and Palaniappan L, Indian J Pure Appl Phys., 2008, 46, 852-856.

4. Thirumaran S and Job Sabu K, Indian J Pure Appl Phys., 2009, 47(2), 87-96.

5. Kannappan V and Jaya Santhi R, Indian J Pure Appl Phys., 2005, 43, 750-754.

6. Kannappan V, Xavier Jesu Raja S and Jaya Santhi R, Indian J Pure Appl Phys., 2003, 41, 690.

7. Jaya Kumar S, Karunanithi N, Kannappan V and Gunasekharan S, Asian J Chem Lett., 1999, 3, 224.

8. $\quad$ Neuman M S and Blum, J Am Soc, 1964, 86, 5600.

9. John A. Dean, Lange, Hand Book of Chemistry, $15^{\text {th }}$ Edn., Mc Graw Hill Inc; New York, 1998.

10. Arul G and Palaniappan L, Indian J Pure Appl Phys., 2005, 43, 755.

11. Eyring H and Kincaid J F, J Chem Phys., 1938, 6, 620.

12. Thirumaran S and Earnest Jaya Kumar J, Indian J Pure Appl Phys., 2009, 47, 265.

13. Mishra A P and Dwivedi D K, Indian J Pure Appl Phys., 2008, 46, 852-856.

14. Thiyagarajan R and Palaniappan L, Indian J Pure Appl Phys., 2008, 46(12), 852-856.

15. Kalaivani $\mathrm{T}$ and Krishnan S, Indian J Pure Appl Phys., 2009, 47, 383-385.

16. Anwar Ali and Anil Kumar Nain, J Pure Appl Ultrasonics., 2000, 22, 10. 


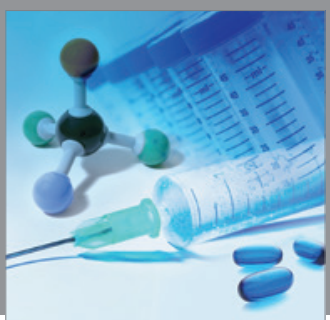

International Journal of

Medicinal Chemistry

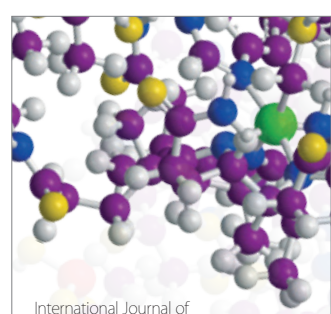

Carbohydrate Chemistry

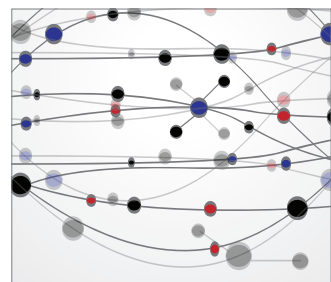

The Scientific World Journal
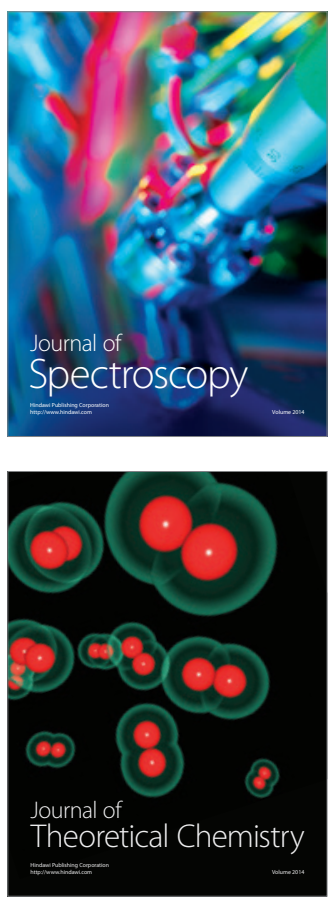
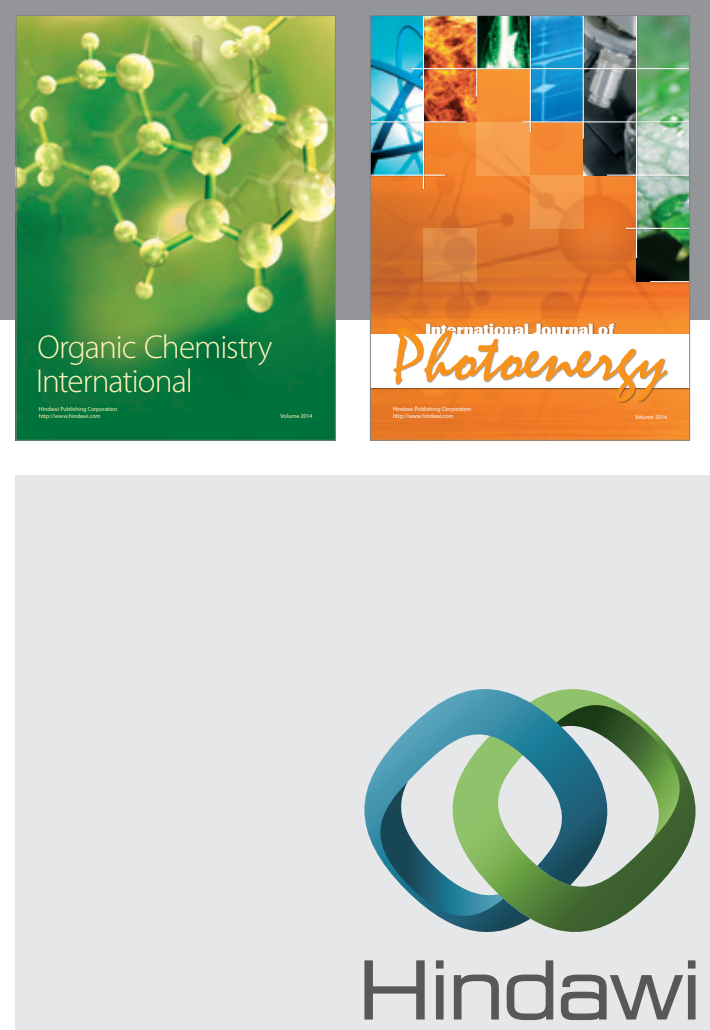

Submit your manuscripts at

http://www.hindawi.com
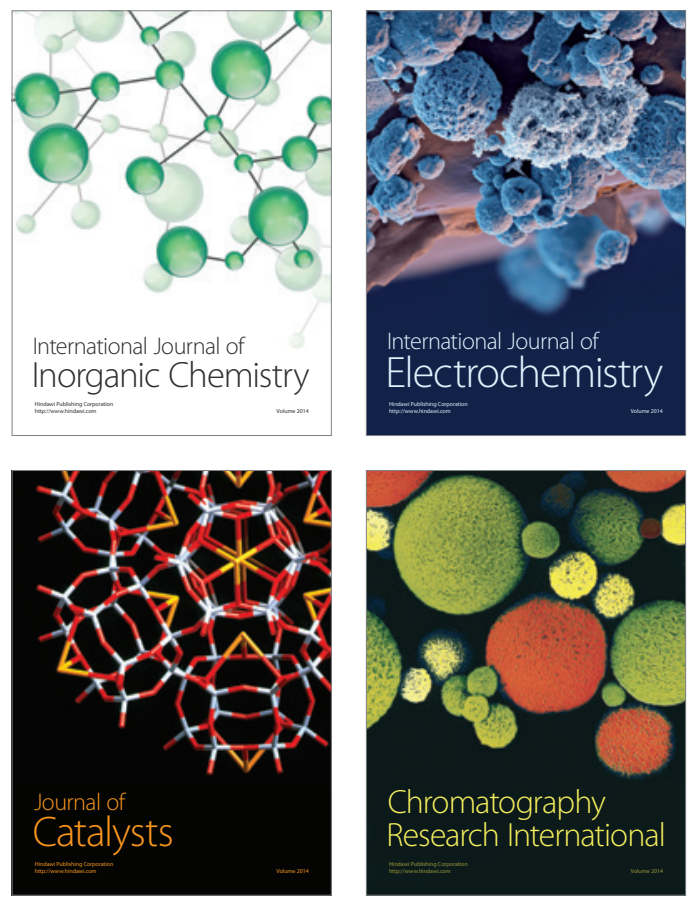
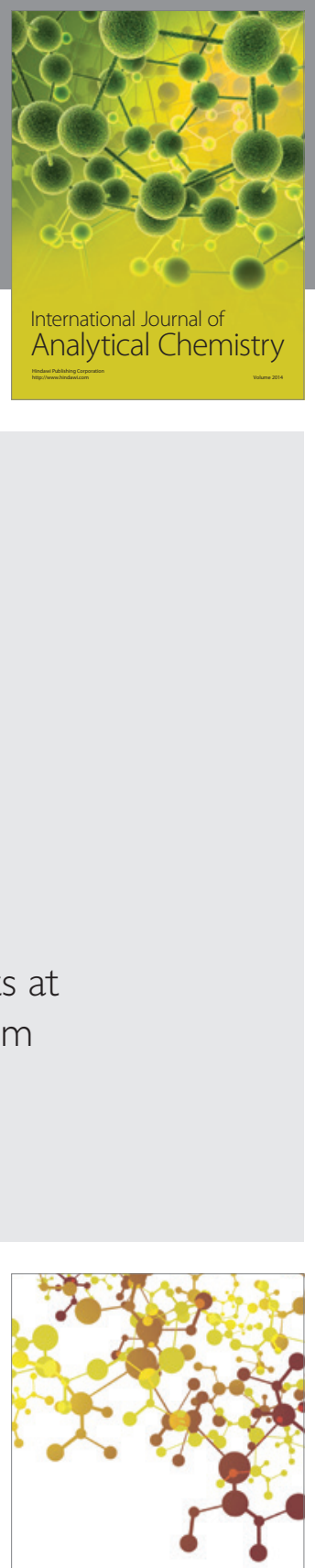

Journal of

Applied Chemistry
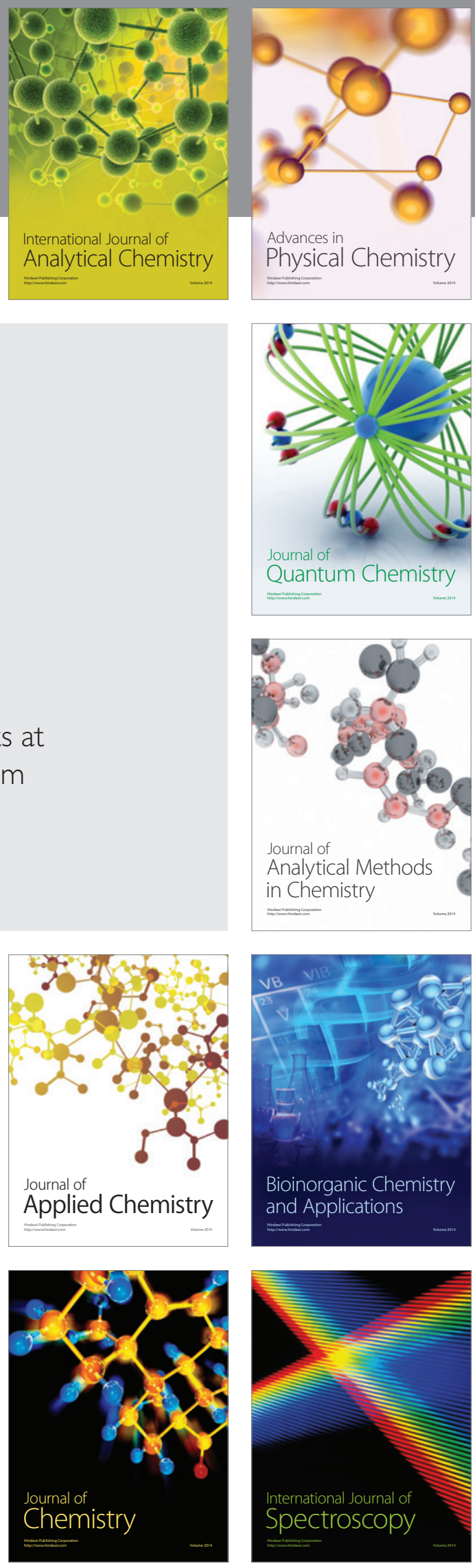